\title{
Barriers, facilitators and recommendations for the early infant diagnosis and treatment (EIDT) cascade: A qualitative study in Malawi
}

\author{
${ }^{1}$ Elizabeth Glaser Pediatric AIDS Foundation, Washington DC, USA \\ ${ }^{2}$ Elizabeth Glaser Pediatric AIDS Foundation, Lilongwe, Malawi \\ ${ }^{3}$ Ministry of Health, Lilongwe, Malawi
}

E A Bobrow, ${ }^{1} \mathrm{PhD}, \mathrm{MPH}$; A G Yemaneberhan, ${ }^{2} \mathrm{MD}, \mathrm{MPH} ; \mathbf{M}$ Phiri, ${ }^{2} \mathrm{MPH}$; L Katirayi, ${ }^{1} \mathrm{MS}$; A Ahimbisibwe, ${ }^{2} \mathrm{MB} \mathrm{ChB}, \mathrm{MPH}$; F Chimbwandira, ${ }^{3} \mathrm{MBBS}, \mathrm{MPH}$; $\mathbf{N}$ Buono, ${ }^{2} \mathrm{MPH}$

Corresponding author: E A Bobrow (ebobrow@pedaids.org)

\begin{abstract}
Background. Identifying and testing all HIV-exposed infants (HEIs) by 2 months of age with rapid antiretroviral therapy (ART) initiation for those infected is critical to survival. Yet in 2009 only $29 \%$ of HEIs in need of ART received treatment in Malawi.

Objectives. To understand barriers, facilitators and recommendations for five key steps in the early infant diagnosis and treatment (EIDT) cascade: (1) identification of HEIs; (2) infant testing; (3) sample processing and transport; (4) reporting results to mothers; (5) ART initiation for HEI.

Methods. Semistructured interviews were conducted through convenience sampling with mothers of infants eligible for EIDT ( $n=47$ ) and with healthcare workers (HCWs) providing EIDT $(n=20)$ in five facilities, in April 2013.

Results. (1) Reliance on the health passport to identify HEIs is both barrier, as women may not attend appointments with their passports, and facilitator, for documentation of HIV-exposure status. Use of trained health surveillance assistants in EIDT enhances cascade steps 1 and 2, but requires increased supervision. (2) Women struggle to accept their own HIV status, yet test results for an HEI is a motivator. Sensitisation through local leadership facilitates EIDT. (3) A reliable transport system is needed. (4) Maintaining appointments to report results to women motivates them. (5) Mothers were reluctant to give ART to young, apparently healthy infants.

Conclusion. Both women and healthcare workers are motivated by test results for HEIs. The inclusion of community education can improve all steps in the EIDT cascade, including information that HEIs need ART.
\end{abstract}

S Afr J Child Health 2016;10(2):116-120. DOI:10.7196/SAJCH.2016v10i2.982

Globally, 3.2 million children under 15 years old were living with HIV in 2013, comprising $9.1 \%$ of all people living with HIV. Of these children, $91 \%$ live in sub-Saharan Africa, and only $24 \%$ were receiving antiretroviral therapy (ART). Without ART, half of the children infected with HIV at birth or in infancy will die before their second birthday, and $80 \%$ will die before their fifth birthday. ${ }^{[1]}$ Although the early diagnosis of HIV in infants is improving in many countries, in 2013 only $42 \%$ of infants born to mothers living with HIV in low- and middle-income countries received HIV testing within 2 months of birth as recommended. ${ }^{[1]}$ Even if children are tested for HIV, not all receive the result or are treated as needed.

Opportunities to optimise infant outcomes may be lost at each step in a cascade of early infant diagnosis and treatment (EIDT) ${ }^{[2]}$ The EIDT cascade includes a number of sequential steps, specifically: (1) identification of HIV-infected infants (HEIs); (2) infant HIV testing; (3) sample processing and transport; (4) reporting results to caregivers; and (5) ART initiation for HEIs.

In Malawi, $13.8 \%$ of HEIs in 2009 were HIV-infected, but only $29 \%$ of those in need of ART received treatment. ${ }^{[3]}$ Studies of antenatal care (ANC), EIDT and paediatric HIV programmes in Blantyre and Lilongwe, Malawi, revealed that there are significant drop-outs at each step of EIDT cascade. ${ }^{[4]}$ Similarly, a Blantyre study identified factors related to failure by women to return for their infant's EID test results. The main barriers were expense due to travel time and costs (adjusted risk ratio (aRR) 1.93; 95\% confidence interval (CI) 1.1 - 3.4), and disclosure by women to their husbands or partners that the infant was being tested (aRR 1.97; 95\% CI: 1.0 - 3.8). ${ }^{[5]}$

Policy changes in Malawi that support ART initiation for HEI include the Paediatric HIV Care Scale-up Plan (2009 - 2013), which authorised all levels of healthcare workers (HCWs) to prescribe ART. However, in practice, paediatric HIV testing, care and treatment were not adequately provided in facilities. Reasons include limited integration of HIV in maternal and paediatric services, lack of social support to women, fear of disclosure of infant HIV status, stigma and lack of knowledge of timing for paediatric testing. ${ }^{[4,6-8]}$ Noted recommendations are to address multiple barriers across the system to improve coordination and to design interventions to increase access to EIDT services, and to ensure that HEIs receive ART soon after diagnosis. ${ }^{[8]}$ With the global target of elimination of motherto-child transmission (eMTCT) ${ }^{[9]}$ adopted by the Government of Malawi, EID of HIV and early initiation of ART are critical for the survival of HEIs and infected children. The goal of this qualitative study was to explore and contribute to an improved understanding of barriers, facilitators and potential solutions in EIDT service delivery at key points throughout the EID cascade.

\section{Methods \\ Design}

Semistructured interviews were conducted with HCWs who were involved with EIDT activities, and with mothers of HEIs. Convenience sampling was utilised to recruit participants. Ethical approval was received from the Malawi National Health Sciences Research Committee (NHSRC).

\section{Settings and participants}

The study was conducted within five purposively selected health facilities within three districts in Malawi that were supported with funding from ViiV-Healthcare to the Elizabeth Glaser Pediatric 
AIDS Foundation (EGPAF). HCWs interviewed included nurses in the ART clinics, ANC and maternity wards, laboratory assistants and health surveillance assistants (HSAs), who are a cadre of trained health outreach workers who work in health facilities and in communities. The HSAs in the selected districts were specifically trained to identify HEIs, to obtain dried blood spot (DBS) samples, and to assist with providing test results plus referral and follow-up for women with HEIs. The HCWs who participated in the study were recruited from antenatal and postnatal clinics as they had received training to identify HEIs, and to provide ART. Mothers eligible for the study included those who had HEIs $<12$ months of age. Women were recruited from the maternal and child health $(\mathrm{MCH})$ clinics. At the time of data collection, many of the health facilities were in the process of using an electronic medical record (EMR) system in the ART clinics to track individuals.

\section{Data collection and analysis}

Data collection occurred in April 2013. All study personnel who interacted with participants or with their data received training on study-specific data collection methods, interview guides and research ethics. All participants provided written informed consent prior to participation. Interviews were conducted individually in a private place. Audio recordings of all interviews were transcribed and translated into English. Transcript files in Microsoft Word (Microsoft Corp., USA) were analysed using MAXqda (Marburg, Germany), a qualitative data analysis software package. Data collection and analysis were structured along the five EIDT cascade steps. The code list and definitions were developed based on research questions. Matrices of coded responses by each participant group for each step in the cascade were developed and compared..$^{[10,11]}$ Themes within each step in the cascade focused on the process of completing each step, the barriers experienced, the facilitators identified, and

Table 1. Barriers and facilitators for each step in the EIDT cascade from study participants ${ }^{*}$

\section{Steps in the EIDT}

cascade Barriers

Step 1: $\quad n=32$

Identification

of HEIs
Women do not bring their health passport, a record of relevant health information, including HIV status, to the health facilities

Lack of follow-up supervision with HSAs who provide EIDT services

Lack of disclosure of HIV status to the partners/husbands

\section{Step 2:}

Infant HIV

testing

Step 3:

Sample

processing

and transport

$n=44$

Lack of space at health centres

Difficulties with obtaining blood specimens from infants for DBS testing

Lack of acceptance of HIV status

Women want to check with their husbands about having the infant tested

$n=30$

No reliable transport system and lack of fuel for specimen transport

Specimens arriving too late at the lab - after the person receiving samples has left for the day

Step 4:

Reporting

results to

caregivers

Step 5:

ART

initiation for

HIV-infected

infants $n=97$

Coming to facility to discover that the results are not available as expected is discouraging - if it happens more than once, unlikely to ever return for results

Unpredictable and irregular return of results

Traveling long distances to the facility is a common complaint cost of transport, difficulty in travel, and long days

Request for privacy so that neighbours do not know your business

Long queues in different rooms for services

$n=29$

Some places have ART clinic only on certain days of the week and if not on day of a woman's $\mathrm{MCH}$ appointment, it is a hardship to make long trip again

It is popularly believed that starting ART in young children will cause complications so people delay getting results or starting ART
Facilitators

$n=54$

Identification of the HEI through the health passport

HSAs have been trained to identify HEIs

Referrals and coordination between the clinics within the

health centre working well

Health education about EIDT in the community is

happening with limited coverage

New PMTCT/ART guidelines have HCWs enrolling HEIs into

HIV care system immediately after delivery to facilitate better tracking

$n=74$

HSAs have been meeting among themselves to reinforce their DBS training and to mentor those with less experience HCWs report more support from village leadership to promote testing and treatment has been important

Women want to protect their infants by knowing their HIV status

$n=52$

Address transport issues by using HSAs on motorbikes, reimbursements for transport, use the ambulance as much as possible

Some sites started regular meetings at the health centre level to discuss issues and solve problems

Some places had a SMS system in place that helped with receiving results

$n=83$

Good coordination between clinics in health centres and to the community

HCWs believe that most women are willing to know the status of their child

Follow-up system at the health centre - motivated by appointments made with women to give them the results Women reinforce belief of HCWs that they feel encouraged and well informed by knowing the HIV result of their infant $n=19$

EID log tracks patients across services/facilities makes follow-ups easier

Testing the infant in good time and having facility nearby Home visits from HCW provide needed support

Women's expectations are changed when ART works and the baby survives 
recommendations for improved service delivery. Questions for the women included motivation for having their infant tested and for collecting the test result, challenges faced when interacting with the health facility and with HCWs, methods for overcoming challenges, and suggestions for improvement. Questions for HCWs followed a similar sequence for each step in the cascade, for example, 'What do you do to overcome challenges?' For two of the steps in the cascade, specifically identification of HEI (step 1) and sample processing and transport (step 3), relevant questions were only asked to HCWs.

\section{Results}

The results are reported on the main barriers, facilitators (Table 1) and recommendations (Table 2) for each cascade step that emerged during analysis. As noted in the tables, relevance of the results is indicated by the order of the themes, with the most frequent responses listed first and subsequent responses listed in descending order. In addition, the results in the text are also in order of importance. Comparisons in responses between mothers and HCWs are noted when relevant.

\section{Demographics}

A total of 47 mothers were interviewed (8 - 10 per site). Their ages ranged from $19-48$ years with an average of 31 years. A total of 41 women $(87 \%)$ were married. Overall, $6(13 \%)$ women had no education, $28(60 \%)$ completed primary education only, $12(25 \%)$ completed secondary education, and 1 (2\%) completed tertiary education. All children received HIV testing; 39 (83\%) mothers knew their child's HIV status and $8(17 \%)$ mothers had not yet received the results. There were 5 HIV-infected children among those with known status, all of whom were initiated on ART. A total of $20 \mathrm{HCWs}$ across service delivery areas were interviewed (10 nurses, 7 HSAs, 2 laboratory assistants, 1 clinical officer).

\section{Step 1: Identification of HEls - responses from HCWs only} Reliance on the health passport, a record of relevant health information including HIV status, was both a barrier and a facilitator to identifying HEIs. Women retain the passport and are asked to bring it to each visit. HCWs rely on this record and on the mother's willingness and memory to bring their passport.
'... some clients are more 'clever'; they hide their health profile ... and get another one or ... they take off a page or change the facility ... but nowadays people are coming out in the open; many are not doing that anymore through sensitisation.' (HCW)

A facilitator centred on the role of the HSAs, who have become an integral part of EIDT. Almost all HSAs at the study sites were trained to identify (step 1) and collect DBS specimens from HEIs (step 2). The need for consistent support and supervision of HSAs was noted.

'... the more they are staying without supervision, the more we are compromising the whole system. If anything, there has to be a regular supervision schedule.' (HCW)

\section{Step 2: Infant HIV testing}

HCWs felt that mothers struggle to accept their own HIV status and therefore have difficulty having their infants tested. Some women are not comfortable to test their children without the knowledge and permission of their husband.

'... they have to go home and explain first to their husbands. If he accepts, they will come back together. But what happens is that when she comes for next visit she gives excuses like "we have not yet discussed it" or "we should wait". (HCW)

Other barriers include difficulties with obtaining blood samples from infants. In some facilities, HSAs reported meeting as a group to reinforce the training they received on taking DBS samples from infants. One of the main facilitators is the support of local leaders to promote HIV testing and treatment for HEIs.

'In the community, the village headmen assert that they would like all HIV-infected women in their villages to get services and that no one needed to be shy about their HIV status.' (HCW)

An aspect of the sensitisation that has been helpful is a focus on the fact that mothers can protect their children by testing them for HIV, thus overcoming the barrier of women accepting their HIV results as a key to good care for their HIV-exposed child. Mothers indicated that knowing the status of their children encouraged them to care properly for their babies.

Table 2. Recommendations for each step in the EIDT cascade from study participants ${ }^{\star}$

Steps in the EIDT cascade Summary of recommendations from participants

Step 1: Identification of HEIs $(n=28)$

\section{Step 2: Infant HIV testing $(n=40)$}

Step 3: Sample processing and transport $(n=39)$

Step 4: Reporting results to caregivers $(n=87)$

Step 5: ART initiation for HEIs $(n=36)$
Educate HIV-infected women on the importance of bringing their health passports to appointments, for the health of their infants

Expand the EMR system, which at the time of data collection only existed for people on ART, to the MCH clinics to enhance and fill gaps in identification of HEIs

Support healthcare workers with additional training related to EIDT related tasks, e.g. taking blood from infants Enhance sensitisation in the communities about HIV testing of infants Address stock-out issues at central supply and within district leadership

Conduct regular supportive supervision of healthcare workers to reinforce skills and knowledge

Provide sensitisation in the communities by engaging the local leadership and highlighting the need for women to have HIV test results for their child for either continued prevention or treatment

Encourage HIV-infected women to talk to other HIV-infected women, possibly one-on-one or in support groups

Establish a lab sample transportation system

Have a transport system for taking specimens to the lab

Systematically follow up on results

Utilise SMS system for reporting results back to the health centre

Enhance counselling on the process for testing and reporting

Counsel women at the health centre and directly in communities

Honour appointments for reporting results or contact women, possibly using mobile phones, to reschedule

Provide education on the need to start ART in HIV-infected children - even if they are young or do not seem sick

${ }^{*}$ Items in the table are listed from most frequently mentioned to least frequent within each step in the cascade. 
'... when you know the HIV status of a child, you give them the much-needed care, and they also start medication right away. When you face any difficulties or challenges, you come to the hospital ... in such a way you prevent the baby from getting infected.' (HIVinfected mother)

\section{Step 3: Sample processing and transport - responses from HCWs only}

The main barrier mentioned was a lack of a reliable transport system from health centres to the central labs where the DBS samples are analysed. An interrelated barrier was the lack of provision at the central lab for leaving DBS samples after hours once the lab has closed. Many sites have tried to address transportation difficulties, including having HSAs take the samples by motorbike, or having a reimbursement system in place for individuals who transport specimens to the central lab. Participants at each study site mentioned how, at the time of data collection, ambulances that are supposed to be available for specimen transport to the central lab are often occupied locally. Having an established system for transport and for the timely communication of results that functions reliably between the health facilities and the central lab was a facilitator. Appointments given to women to receive test results for their HEIs (step 4) should serve as motivators, as these serve as deadlines for test results to be available from the central labs. Some sites have established regular meetings with HCWs to discuss EIDT issues as they arise, to solve problems as a team, and to remind them to schedule appointments with the mothers to receive test EIDT results. In some places, mobile phone SMS messages have been used to transmit test results from the central lab to either the district lab or the health facility.

\section{Step 4: Reporting results to mothers}

The primary barrier identified by both HCWs and mothers was that health facilities fail to have the EID results ready when women return for scheduled appointments. Mothers reported spending money on transport and wasting their time unnecessarily to return to the facility.

'I waited too long without getting the results. I came five times and found no results. So you can imagine that we use money for transport and for someone to be coming and finding nothing. It's frustrating.' (HIV-infected mother)

Health centres needs to honour the appointments given to women for their infant's result. Appointments scheduled during the DBS sample collection in step 2 raise expectations. HCWs generally felt that women are willing to know the status of their child even if they are fearful, which was echoed by the women.

'I wanted to be a free person, not worrying about his health and seeing him suffer.' (HIV-infected mother)

'You feel comfortable, and also if the baby is not okay, you know how best to take care of him.' (HIV-infected mother)

\section{Step 5: ART initiation for HEI}

One main barrier noted was that at many facilities, ART clinics only occur on certain days of the week and thus women have to return to the health centre on multiple days for various types of care. At the study sites, HEIs are initiated on ART and receive follow-up care in ART clinics only. HCWs also report that women often believe that ART is not appropriate for infants who are young and healthy, a belief that is a barrier to women initiating their HEI on ART. As one healthcare worker explained:

'The perception they have is that you can only be tested or start ART when you are sick. Thus, the people's mindsets are something to be dealt with. Otherwise, one cannot force them to do the tests.'

At the facility, a number of interventions have improved initiation of ART for infants, specifically the use of EID log books to track patients, having a nearby ART clinic for children, and enhanced counselling.

\section{Recommendations from participants for each step in the EIDT cascade}

Table 2 presents a summary of participant recommendations for each step in the EIDT cascade. The results are listed from most frequent responses to least frequent for each step in the cascade. For the identification of HEIs in step 1, HCWs suggested enhanced messages directed to women, specifically emphasising the importance of bringing their health passports to each visit, and of disclosing their HIV status to their partners. HCWs recommended expanding the existing EMR system, which at the time of data collection was only used for patients on ART. In the community, they recommended enhancing sensitisation about HIV testing of infants. For sample collection in step 2, recommendations included promoting community sensitisation through local leadership with an emphasis on prevention and treatment once a mother knows the HIV status of her child. In addition, HCWs strongly suggested higher-quality, comprehensive counselling at the facility, including encouragement for women to talk to other HIV-infected women. Improved counselling could increase the demand for EIDT services and improve the acceptability of the EIDT services within the community. For sample processing and transport in step 3, the HCWs recommended the need for reliable, designated transport system for taking specimens to the lab, systematic follow-up for all results, use of electronic data management systems, utilisation of SMS to report results back to the health centres, regular follow-up with the lab, and sending a district-based or facility-based lab person with the samples or at least providing orientation to drivers delivering samples. For reporting results to mothers in step 4, HCWs recommended setting realistic expectations for the arrival of DNA-PCR results. Airtime for mobile phones was recommended by both the HCWs and the mothers. As a mother said: 'They should call us to tell us the results since we come from far distances.' For ART initiation of HEIs in step 5, HCWs suggested education in the community and comprehensive counselling in the facility on the need and benefits of initiating HIVinfected children on ART, even if they are young or do not seem sick.

\section{Discussion}

Women are motivated by receiving the test results for their HEIs HCWs agree that improving the system to increase the number of HEIs tested and the number of women receiving results is critical. For every step in the EIDT cascade, the inclusion of community education is essential, particularly including information that all HEIs need ART regardless of their age and health status.

The study highlights specific actions to enhance steps along the EIDT cascade. The identification of HEIs (step 1) may benefit from an enhanced tracking system of HEIs, either through a paper-based system throughout the health facility or, as stated as a recommendation, through adding key data related to EIDT for the entire cascade to the existing EMR system, which at the time of the data collection was only utilised to track individuals on ART. Expanding the EMR system, thus decreasing the reliance on health passports that was identified as both a barrier and facilitator in step 1, may improve follow-up with women and their HEIs through the time of testing (step 2) and obtaining results (step 4).

Increasing testing of HEIs (step 2) needs community-based support. Connecting women, either individually or in support groups, can address the identified barriers of reluctance to disclose to husbands. Another study focused on EIDT recommended sensitisation campaigns through radio and advertisements to increase uptake of EID. ${ }^{[12]}$

Sample processing and transport (step 3) results indicate the need for the establishment of a strong sample transport system and a laboratory information system possibly using SMS. ${ }^{[13]}$ In addition, having procedures that work for after-hours sample delivery and for reporting of results would address barriers. Another study encourages 
initiation with point-of-care testing as the best solution to avoid issues of sample transport and hence fast delivery of results. ${ }^{[14]}$

Recommendations indicate that if an appointment is scheduled with a woman to receive test results (step 4), it either needs to be kept or the woman needs to be contacted to reschedule the appointment. One study found that HIV-infected mothers enrolled in HIV care were less likely to fail to return for their infant's HIV test result (aRR $0.51 ; 95 \% \mathrm{CI}(0.3-0.9),{ }^{[5]}$ therefore providing ART to all pregnant women may lead to a better return. This is encouraging for Malawi, which is implementing lifelong ART for all HIV-positive pregnant women regardless of CD4 count.

ART initiation for HEIs (step 5) may benefit from an emphasis on interactions with other women, on counselling from HCWs and on community-level sensitisation from HSAs and local leaders on how children improve and live longer lives when on treatment. A study in South Africa found similar results related to how support of women, specifically by seeing health benefits of children on treatment, was a motivating factor for improved EIDT. ${ }^{[15]}$ These peer-to-peer connections were also mentioned as a method to improve testing for HIV-exposed child (step 2).

More training for HSAs and other HCWs would enhance multiple steps in the EIDT cascade. Training topics could include techniques to communicate with women about the importance of their health passports (step 1), to successfully take blood from infants for EID tests (step 2), to help women accept their HIV status and to have their HEIs tested (step 2), to follow-up with leaders in the facility to address stock-outs (step 1) and to give feedback on the transport system (step 3), to counsel mothers about putting HEIs on ART once they test HIV-positive (step 5). Increased supervision would be needed as follow-up to enhanced training.

One strength of this study is that while other studies have reported decreases in the number of infants moving through the EIDT cascade,$^{[3,4]}$ and on barriers and facilitators to EIDT in general, ${ }^{[12,15]}$ previous studies have not focused on distinct motivators, challenges and solutions for each step along the EIDT cascade. Limitations include that we only interviewed women who had their HIV-exposed child tested and were at the health facility for the result. The study also only included women with HIV-infected children who initiated ART. In addition, voices of mothers were limited to only three of the five steps in the cascade in the study.

\section{Conclusion}

Findings from this study provide relevant and important information to improve service delivery and to increase uptake of EIDT along five key steps in the EIDT cascade. The study also expands on current literature by assessing HCWs' and mothers' perspectives on barriers and facilitators of EIDT. The findings have implications for future interventions at the health facilities and in the community for HCWs and for mothers to ensure that EIDT operates optimally for the survival of HIV-exposed and -infected children.
Acknowledgements. The authors wish to thank all the healthcare workers and mothers who participated in this study, and the staff members in the EGPAF Malawi and US offices who provided technical and programmatic support for carrying out the study. We acknowledge the Ministry of Health, HIV/AIDS Department, and the District Offices of Dedza, Ntcheu, and Lilongwe for their collaboration and support of the study. This study was funded by ViiV Healthcare.

\section{References}

1. Joint United Nations Programme on HIV/AIDS (UNAIDS). The Gap Report. 2014. http://www.unaids.org/sites/default/files/en/media/unaids/ contentassets/documents/unaidspublication/2014/UNAIDS_Gap_report en.pdf (accessed 1 December 2015).

2. Ciaranello AL, Park JE, Ramirez-Avila L, et al. Early infant HIV-1 diagnosis programs in resource-limited settings: Opportunities for improved outcomes and more cost-effective interventions. BMC Med 2011;9:59. DOI:10.1186/1741-7015-9-59

3. Dube Q, Dow A, Chirambo C, et al. Implementing early infant diagnosis of HIV infection at the primary care level: Experiences and challenges in Malawi. Bull World Health Organ 2012; 90(9):699-704. DOI:10.2471/BLT.11.100776

4. Braun M, Kabue MM, McCollum ED, et al. Inadequate coordination of maternal and infant HIV services detrimentally affects early infant diagnosis outcomes in Lilongwe. J Acquir Immune Defic Syndr 2011;56(5):e122-128. DOI:10.1097/QAI.0b013e31820a7f2f

5. Cromwell EA, Dow AE, Low D, et al. Barriers to successful early infant diagnosis of HIV infection at primary care level in Malawi. Pediatr Infect Dis J 2015;34(3):273-275. DOI:10.1097/inf.0000000000000625

6. Adeniyi VO, Thomson E, Ter Goon D, Ajayi IA. Disclosure, stigma of HIV positive child and access to early infant diagnosis in the rural communities of OR Tambo District, South Africa: A qualitative exploration of maternal perspective. BMC Pediatr 2015;15:98. DOI:10.1186/s12887-015-0414-8

7. Cherutich P, Inwani I, Nduati R, Mbori-Ngacha D. Optimizing paediatric HIV care in Kenya: Challenges in early infant diagnosis. Bull World Health Organ 2008;86(2):155-160. DOI:10.2471/blt.07.040402

8. Feucht UD, Meyer A, Thomas WN, Forsyth BW, Kruger M. Early diagnosis is critical to ensure good outcomes in HIV-infected children: Outlining barriers to care. AIDS Care 2016;28(1):32-42. DOI:10.1080/09540121.2015.1066748

9. Joint United Nations Programme on HIV/AIDS (UNAIDS). Countdown to Zero: Global Plan Towards the Elimination of New HIV Infections Among Children by 2015 and Keeping Their Mothers Alive, 2011 2015. http://files.unaids.org/en/media/unaids/contentassets/documents/ unaidspublication/2011/20110609_JC2137_Global-Plan-Elimination-HIVChildren_en.pdf (accessed 1 December 2015).

10. Miles MB, Huberman AM. Qualitative Data Analysis. London: Sage Publications, 1994:325.

11. Ulin P, Robinson E, Tolley E. Qualitative Methods in Public Health: A Field Guide for Applied Research. San Fransisco: Jossey-Bass, 2005.

12. Boender TS, Sigaloff KC, Kayiwa J, et al. Barriers to initiation of pediatric HIV treatment in Uganda: A mixed-method study. AIDS Res Treat 2012;2012:817506. DOI:10.1155/2012/817506

13. Finocchario-Kessler S, Gautney BJ, Khamadi S, et al. If you text them, they will come: Using the HIV infant tracking system to improve early infant diagnosis quality and retention in Kenya. AIDS 2014;28(3):S313-321. DOI:10.1097/ QAD.0000000000000332.

14. Anderson DA, Crowe SM, Garcia M. Point-of-care testing. Curr HIV/AIDS Rep 2011;8(1):31-37. DOI:10.1007/s11904-010-0067-z

15. Yeap AD, Hamilton R, Charalambous $S$, et al. Factors influencing uptake of HIV care and treatment among children in South Africa - a qualitative study of caregivers and clinic staff. AIDS Care 2010;22(9):1101-1107. DOI:10.1080/09540121003602218 